\title{
LARGE BENT JETS IN THE INNER REGION OF CSS
}

\author{
F. MANTOVANI ${ }^{1}$, W. JUNOR ${ }^{2}$, M. BONDI ${ }^{1}$, L. PADRIELLI ${ }^{1}$, \\ W. COTTON ${ }^{3}$ AND E. SALERNO ${ }^{1}$ \\ 1 Istituto di Radioastronomia, Bologna, Italy \\ 2 Dept. of Astrophysycs, University of New Mexico, USA \\ ${ }^{3}$ National Radio Astronomy Observatory, Charlottesville, USA
}

\section{Introduction}

Recently we focussed our attention on a sample of Compact Steep-spectrum Sources (CSSs) selected because of the large bent radio jets seen in the inner region of emission. The largest distortions are often seen in sources dominated by jets, and there are suggestions that this might to some extent be due to projection effects. However, superluminal motion is rare in CSSs. The only case we know of so far is $3 \mathrm{C} 147$ (Alef at al. 1990) with a mildly superluminal speed of $\sim 1.3 v / c$. Moreover, the core fractional luminosity in CSSs is $\sim 3 \%$ and $\leq 0.4 \%$ for quasars and radio galaxies respectively. Similar values are found for large size radio sources i.e. both boosting and orientations in the sky are similar for the two classes of objects. An alternative possibility is that these bent-jet sources might also be brightened by interactions with the ambient media. There are clear indications that intrinsic distortions due to interactions with a dense inhomogeneous gaseous environment play an important role. Observational support comes from the large RMs found in CSSs (Taylor et al. 1992; Mantovani et al. 1994; Junor et al. these proc.) and often associated with strong depolarization (Garrington \& Akujor, t.p.). The CSSs also have very luminous Narrow Line Regions emission, with exceptional velocity structure (Gelderman, t.p.).

\section{Our investigation}

We are investigating a sample of sources showing large bent jets on the mas scale. Table 1 lists the selected sources. It is possible that these are extreme 
TABLE 1. Source parameters.

\begin{tabular}{|c|c|c|c|c|c|c|c|}
\hline Source & ref & $\begin{array}{l}\text { dist } \\
\text { mas }\end{array}$ & $\begin{array}{r}\text { dist } \\
\text { pc }\end{array}$ & $\begin{array}{r}\Delta P A \\
\operatorname{deg}\end{array}$ & $\mathbf{z}$ & O.I. & $\begin{array}{r}\mathrm{RM} \times(1+z)^{2} \\
\quad \mathrm{rad} \mathrm{m}^{-2}\end{array}$ \\
\hline $0127+2333 \mathrm{C} 43$ & a & 225 & 780 & 93 & 1.459 & $\mathbf{Q}$ & -1088 \\
\hline $0358+0043 \mathrm{C} 99$ & b & 15 & 46 & 60 & 0.425 & G & 40 \\
\hline $0429+4153 \mathrm{C}_{119}$ & c & 39 & 142 & 55 & 1.023 & $\mathbf{Q}$ & 3400 \\
\hline $0538+4983 C_{147}$ & d & 200 & 664 & 90 & 0.545 & $\mathbf{Q}$ & $-3222 / 621$ \\
\hline $0548+165$ & $\bullet$ & 80 & 253 & 90 & 0.474 & $\mathbf{Q}$ & 1934 \\
\hline $1328+2543 \mathrm{C} 287$ & $\mathbf{f}$ & & & 58 & 1.055 & $\mathbf{Q}$ & - \\
\hline $1442+101 O Q 172$ & $\mathbf{g}$ & 15 & 39 & 90 & 3.531 & $\mathbf{Q}$ & 22400 \\
\hline $1629+6804 C 68.18$ & $\mathrm{~h}$ & 76 & 226 & 66 & 2.475 & $\mathbf{Q}$ & - \\
\hline $1741+279 B 2$ & - & 300 & 862 & 84 & 0.372 & $\mathbf{Q}$ & $-219 / 293$ \\
\hline $2033+187$ & - & 40 & 129 & 80 & $(0.5)$ & - & $\mathrm{r}$ \\
\hline $2147+145$ & • & 23 & 75 & 90 & $(0.5)$ & - & $\mathrm{r}$ \\
\hline
\end{tabular}

Note: In column 8 , the mark - means no measurements available, the mark $n$ means polarization not detected. References: a - Spencer at al. (1991); b - Mantovani et al. (1992); c - Nan Ren-dong et al. (1991); d - Alef et al. (1990); e - this investigation; f - Fanti et al. (1989); g - Dallacasa et al. (1995); h - Dallacasa et al. (in preparation)

cases. Features common to these sources are: (a) - a core-jet structure; (b) - a change in the jet major axis Position Angle $\Delta P A>50^{\circ}$; (c) - a bend that occur at a linear separation which is $<1 \mathrm{kpc}$ from the core.

Their mas scale structures lend support for the view of a strong interaction between the jet flow and dense gas clouds. Norman \& Balsara (1993) have investigated the physics of a jet/cloud collision with 3-D hydrodynamical simulations. Their work reveals interesting features that further support this interpretation. For example, the reflected jet inherits the stability properties of the original jet. Nevertheless, the images of sources like $3 \mathrm{C} 119,0548+165$ and $3 \mathrm{C} 287$ represent a puzzle since they would require more than one collision to produce structures that are spiral-like or bent by about $180^{\circ}$.

\section{References}

Alef, W. et al. (1990) Proceedings CSS \& GPS Radio Sources Workshop held in Dwingeloo, ed. by C. Fanti, R.Fanti, C.P. O'Dea and R.T. Schilizzi

Dallacasa, D. et al. (1995) $A \& A$ 295, pp. 27

Fanti, C. et al. (1989) $A \& A$ 217, pp. 44

Mantovani, F. et al. (1994) $A \& A$ 292, pp. 59

Nan Ren-dong et al. (1991) $A \& A$ 245, pp. 449

Norman, M.L. \& Balsara D.S. (1993) Jet in Extragalactic Radio Sources ed. by K. Meisenheimer \& H.J. Roeser, Berlin, Springer Verlag

Spencer R. S. et al. (1991) MNRAS 250, pp. 225

Taylor, G. B., Inoue, M. and Tabara, H. (1992) A\&A 264, pp. 421 